1 Hacettepe Journal of Mathematics and Statistics

$\bigcap$ Volume $44(5)$ (2015), $1045-1055$

\title{
On some new converses of the Jensen and the Lah-Ribarič operator inequality
}

\author{
Rozarija Jakšíć ${ }^{*}$, Mario Krnić ${ }^{\dagger}$ and Josip Pečarić ${ }^{\ddagger}$
}

\begin{abstract}
In this paper we study some new converses of the Jensen and the LahRibarič operator inequality regarding convex functions. First we give two series of converses in a general setting. The general results are then applied to quasi-arithmetic operator means with a particular emphasis to power operator means. The obtained results are also compared with some related results, known from the literature.
\end{abstract}

Keywords: Jensen operator inequality, Lah-Ribarič operator inequality, convexity, operator convexity, converse

2000 AMS Classification: Primary 47A63, 47A64.

Received 24/09/2013 : Accepted 21/01/2014 Doi : 10.15672/HJMS.2015449089

\section{Introduction}

The Jensen inequality is one of the most important inequalities in modern mathematics since it implies the whole series of other classical inequalities (e.g. those by Hölder, Minkowski, Beckenbach-Dresher, Young, the arithmetic-geometric mean inequality etc.). Applications of this inequality in various branches of mathematics, especially in mathematical analysis and statistics, have certainly contributed to its importance. During decades, the Jensen inequality was extensively studied by some famous authors and was generalized in numerous directions. For a comprehensive inspection of the Jensen inequality including history, proofs and diverse applications, the reader is referred to [10].

In this paper we refer to a quite general operator form of the Jensen inequality. In order to present such result, we first introduce the appropriate setting.

*University of Zagreb, Faculty of Textile Technology, Prilaz baruna Filipovića 28a, 10000 Zagreb, CROATIA

Email: jaksic.rozarija@gmail.com

${ }^{\dagger}$ University of Zagreb, Faculty of Electrical Engineering and Computing, Unska 3, 10000 Zagreb, CROATIA

Email:mario.krnic@fer.hr

¥University of Zagreb, Faculty of Textile Technology, Prilaz baruna Filipovića 28a, 10000 Zagreb, CROATIA

Email:pecaric@element.hr 
Let $T$ be a locally compact Hausdorff space and let $\mathcal{A}$ be a $C^{*}$-algebra. We say that a field $\left(x_{t}\right)_{t \in T}$ of elements in $\mathcal{A}$ is continuous if the function $t \rightarrow x_{t}$ is norm continuous on $T$. Additionally, if $T$ is equipped with a Radon measure $\mu$ and the function $t \rightarrow\left\|x_{t}\right\|$ is integrable, then, the so-called Bochner integral $\int_{T} x_{t} d \mu(t)$ can be formed. More precisely, the Bochner integral is the unique element in $\mathcal{A}$ such that the relation

$$
\varphi\left(\int_{T} x_{t} d \mu(t)\right)=\int_{T} \varphi\left(x_{t}\right) d \mu(t)
$$

holds for every linear functional $\varphi$ in the norm dual $\mathcal{A}^{*}$ (see [5]).

Assume furthermore that there is a field $\left(\phi_{t}\right)_{t \in T}$ of positive linear mappings $\phi_{t}: \mathcal{A} \rightarrow \mathcal{B}$ from $\mathcal{A}$ to another $C^{*}$-algebra $\mathcal{B}$. Such field is said to be continuous if the function $t \rightarrow \phi_{t}(x)$ is continuous for every $x \in \mathcal{A}$. If the $C^{*}$-algebras are unital and the field $t \rightarrow \phi_{t}(\mathbf{1})$ is integrable with integral $\mathbf{1}$, we say that $\left(\phi_{t}\right)_{t \in T}$ is unital. We assume that such field is continuous.

If $f: I \rightarrow \mathbb{R}$ is operator convex function, where $I$ is a real interval of any type, and $\left(\phi_{t}\right)_{t \in T}$ is a unital field, then the Jensen operator inequality (see Hansen et.al., [6]) asserts that

$$
f\left(\int_{T} \phi_{t}\left(x_{t}\right) d \mu(t)\right) \leq \int_{T} \phi_{t}\left(f\left(x_{t}\right)\right) d \mu(t)
$$

holds for every bounded continuous field $\left(x_{t}\right)_{t \in T}$ of self-adjoint elements in $\mathcal{A}$ with spectra contained in $I$. If $f: I \rightarrow \mathbb{R}$ is operator concave function, then the sign of inequality in (1.1) is reversed.

Observe that the above inequality refers to an operator convex function. Recall that a continuous function $f: I \rightarrow \mathbb{R}$ is operator convex if

$$
f(\lambda x+(1-\lambda) y) \leq \lambda f(x)+(1-\lambda) f(y)
$$

holds for each $\lambda \in[0,1]$ and every pair of self-adjoint operators $x$ and $y$ (acting) on an infinite dimensional Hilbert space $\mathcal{H}$ with spectra in $I$ (the ordering is defined by setting $x \leq y$ if $y-x$ is positive semi-definite).

In the same paper, Hansen et.al. obtained the following inequality which holds for an usual convex function $f:[m, M] \rightarrow \mathbb{R}$ (see [6], proof of Theorem 2):

$$
\int_{T} \phi_{t}\left(f\left(x_{t}\right)\right) d \mu(t) \leq \alpha_{f} \int_{T} \phi_{t}\left(x_{t}\right) d \mu(t)+\beta_{f} \mathbf{1} .
$$

In this matter, the usual notation is used:

$$
\alpha_{f}=\frac{f(M)-f(m)}{M-m} \text { and } \quad \beta_{f}=\frac{M f(m)-m f(M)}{M-m} .
$$

Inequality (1.2) will be referred to as the Lah-Ribarič operator inequality. Observe that the operator inequality (1.2) is established by applying the functional calculus to the well-known inequality

$$
f(t) \leq \alpha_{f} t+\beta_{t},
$$

which holds for every convex function on the interval $[m, M]$. Recall that $l(t)=\alpha_{f} t+\beta_{t}$ is the linear function limiting convex function $f(t)$ on interval $[m, M]$ from the above.

The main objective of this paper is to derive converses of the above inequalities (1.1) and (1.2). Although inequality (1.1) holds for an operator convex function, both series of converses will be established for convex functions in the classical real sense.

The paper is organized in the following way: after this Introduction, in Section 2 we derive our main results, that is, we obtain two series of converses that correspond to the Jensen and the Lah-Ribarič operator inequality. Further, in Sections 3 and 4 general results are then applied to quasi-arithmetic operator means, with a particular 
emphasis to power operator means. In such a way, we obtain converse inequalities for quasi-arithmetic and power operator means.

The techniques that will be used in the proofs are mainly based on the classical real and functional calculus, especially on the well-known monotonicity principle for self-adjoint elements of a $C^{*}$-algebra $\mathcal{A}$ : If $x \in \mathcal{A}$ with a spectra $\operatorname{Sp}(x)$, then

$$
f(t) \geq g(t), t \in \operatorname{Sp}(x) \quad \Longrightarrow \quad f(x) \geq g(x),
$$

where $f$ and $g$ are real valued continuous functions.

\section{Basic results}

In this section we give our main results, that is, converses of the Jensen and the LahRibarič operator inequality in a general setting presented in the Introduction. As we have already discussed, the results that follow refer to an usual convex function. Although regarding different inequalities, it appears that these two series of converses are closely connected.

First we give a series of converses for the Jensen operator inequality. It should be noticed here that the following theorem in the classical real case was proved by Dragomir in the recent paper [2]. In fact, such series of scalar inequalities will be exploited in establishing the corresponding operator form.

2.1. Theorem. Let $f: I \rightarrow \mathbb{R}$ be a continuous convex function, and let $m, M \in \mathbb{R}$, $m<M$, be such that interval $[m, M]$ belongs to the interior of interval $I$. Further, suppose $\mathcal{A}$ and $\mathcal{B}$ are unital $C^{*}$-algebras, and $\left(\phi_{t}\right)_{t \in T}$ is a unital field of positive linear mappings $\phi_{t}: \mathcal{A} \rightarrow \mathcal{B}$ defined on a locally compact Hausdorff space $T$ with a bounded Radon measure $\mu$. Then the series of inequalities

$$
\begin{aligned}
& \int_{T} \phi_{t}\left(f\left(x_{t}\right)\right) d \mu(t)-f\left(\int_{T} \phi_{t}\left(x_{t}\right) d \mu(t)\right) \\
\leq & \frac{f_{-}^{\prime}(M)-f_{+}^{\prime}(m)}{M-m}\left(M \mathbf{1}-\int_{T} \phi_{t}\left(x_{t}\right) d \mu(t)\right)\left(\int_{T} \phi_{t}\left(x_{t}\right) d \mu(t)-m \mathbf{1}\right) \\
\leq & \frac{1}{4}(M-m)\left(f_{-}^{\prime}(M)-f_{+}^{\prime}(m)\right) \mathbf{1}
\end{aligned}
$$

holds for every bounded continuous field $\left(x_{t}\right)_{t \in T}$ of self-adjoint elements in $\mathcal{A}$ with spectra contained in $[m, M]$. If $f$ is concave on $I$, then the signs of inequalities in (2.1) are reversed.

Proof. Taking into account the operator version of the Lah-Ribarič inequality (1.2), it follows that

$$
\begin{aligned}
& \int_{T} \phi_{t}\left(f\left(x_{t}\right)\right) d \mu(t)-f\left(\int_{T} \phi_{t}\left(x_{t}\right) d \mu(t)\right) \\
\leq & \alpha_{f} \int_{T} \phi_{t}\left(x_{t}\right) d \mu(t)+\beta_{f} \mathbf{1}-f\left(\int_{T} \phi_{t}\left(x_{t}\right) d \mu(t)\right) .
\end{aligned}
$$

On the other hand, regarding convexity of $f$, we have the so-called gradient inequality,

$$
f(t)-f(M) \geq f_{-}^{\prime}(M)(t-M),
$$

which holds for every $t \in[m, M]$, that is,

$$
(t-m) f(t)-(t-m) f(M) \geq f_{-}^{\prime}(M)(t-M)(t-m), \quad t \in[m, M],
$$

after multiplying with $t-m$. In the same way, it follows that

$$
(M-t) f(t)-(M-t) f(m) \geq f_{+}^{\prime}(m)(M-t)(t-m), \quad t \in[m, M] .
$$


Now, adding the above two inequalities, and then, dividing by $m-M$, we have

$$
\alpha_{f} t+\beta_{f}-f(t) \leq \frac{f_{-}^{\prime}(M)-f_{+}^{\prime}(m)}{M-m}(M-t)(t-m) .
$$

Moreover, taking into account the arithmetic-geometric mean inequality, the following series of inequalities holds for all $t \in[m, M]$ (see also [2]):

$$
\begin{aligned}
\alpha_{f} t+\beta_{f}-f(t) & \leq \frac{f_{-}^{\prime}(M)-f_{+}^{\prime}(m)}{M-m}(M-t)(t-m) \\
& \leq \frac{1}{4}(M-m)\left(f_{-}^{\prime}(M)-f_{+}^{\prime}(m)\right) .
\end{aligned}
$$

Now, since $m \mathbf{1} \leq x_{t} \leq M \mathbf{1}$ for every $t \in T$, it follows that $m \phi_{t}(\mathbf{1}) \leq \phi_{t}\left(x_{t}\right) \leq M \phi_{t}(\mathbf{1})$, that is, $m \mathbf{1} \leq \int_{T} \phi_{t}\left(x_{t}\right) d \mu(t) \leq M \mathbf{1}$. Hence, applying the functional calculus to the above series of inequalities, that is, setting $\int_{T} \phi_{t}\left(x_{t}\right) d \mu(t)$ instead of $t$, we have

$$
\begin{aligned}
& \alpha_{f} \int_{T} \phi_{t}\left(x_{t}\right) d \mu(t)+\beta_{f} \mathbf{1}-f\left(\int_{T} \phi_{t}\left(x_{t}\right) d \mu(t)\right) \\
\leq & \frac{f_{-}^{\prime}(M)-f_{+}^{\prime}(m)}{M-m}\left(M \mathbf{1}-\int_{T} \phi_{t}\left(x_{t}\right) d \mu(t)\right)\left(\int_{T} \phi_{t}\left(x_{t}\right) d \mu(t)-m \mathbf{1}\right) \\
\leq & \frac{1}{4}(M-m)\left(f_{-}^{\prime}(M)-f_{+}^{\prime}(m)\right) \mathbf{1} .
\end{aligned}
$$

Finally, comparing (2.2) and (2.5), we obtain (2.1), as claimed.

2.2. Remark. Observe that in the statement of Theorem 2.1 the interval $[m, M]$ belongs to the interior of the interval $I$. This condition assures finiteness of the one-sided derivatives in (2.1). Without this assumption these derivatives might be infinite.

2.3. Remark. It should be noticed here that the first expression in the series of inequalities (2.1), that is, the element $\int_{T} \phi_{t}\left(f\left(x_{t}\right)\right) d \mu(t)-f\left(\int_{T} \phi_{t}\left(x_{t}\right) d \mu(t)\right)$ is not positive in general. This element is positive if $f$ is in addition operator convex function, due to the Jensen operator inequality (1.1).

The following result represents converses of the Lah-Ribarič operator inequality (1.2):

2.4. Theorem. Suppose $f: I \rightarrow \mathbb{R}$ is a continuous convex function, and $m, M \in \mathbb{R}$, $m<M$, are such that interval $[m, M]$ belongs to the interior of interval $I$. Further, let $\left(\phi_{t}\right)_{t \in T}$ be a unital field of positive linear mappings $\phi_{t}: \mathcal{A} \rightarrow \mathcal{B}$, where $\mathcal{A}$ and $\mathcal{B}$ are unital $C^{*}$-algebras, defined on a locally compact Hausdorff space $T$ with a bounded Radon measure $\mu$. Then the series of inequalities

$$
\begin{aligned}
0 & \leq \alpha_{f} \int_{T} \phi_{t}\left(x_{t}\right) d \mu(t)+\beta_{f} \mathbf{1}-\int_{T} \phi_{t}\left(f\left(x_{t}\right)\right) d \mu(t) \\
& \leq \frac{f_{-}^{\prime}(M)-f_{+}^{\prime}(m)}{M-m} \int_{T} \phi_{t}\left(\left[M \mathbf{1}-x_{t}\right]\left[x_{t}-m \mathbf{1}\right]\right) d \mu(t) \\
& \leq \frac{f_{-}^{\prime}(M)-f_{+}^{\prime}(m)}{M-m}\left(M \mathbf{1}-\int_{T} \phi_{t}\left(x_{t}\right) d \mu(t)\right)\left(\int_{T} \phi_{t}\left(x_{t}\right) d \mu(t)-m \mathbf{1}\right) \\
& \leq \frac{1}{4}(M-m)\left(f_{-}^{\prime}(M)-f_{+}^{\prime}(m)\right) \mathbf{1}
\end{aligned}
$$

holds for every bounded continuous field $\left(x_{t}\right)_{t \in T}$ of self-adjoint elements in $\mathcal{A}$ with spectra contained in $[m, M]$. If $f$ is concave on $I$, then the signs of inequalities in (2.6) are reversed. 
Proof. The first inequality in (2.6) holds by virtue of the Lah-Ribarič inequality (1.2). Further, starting from the scalar inequality (2.3), it follows that relation

$$
\alpha_{f} x_{t}+\beta_{f} \mathbf{1}-f\left(x_{t}\right) \leq \frac{f_{-}^{\prime}(M)-f_{+}^{\prime}(m)}{M-m}\left(M \mathbf{1}-x_{t}\right)\left(x_{t}-m \mathbf{1}\right)
$$

holds for every $t \in T$. Now, applying the positive linear mappings $\phi_{t}$ to the above relation, we obtain

$$
\alpha_{f} \phi_{t}\left(x_{t}\right)+\beta_{f} \phi_{t}(\mathbf{1})-\phi_{t}\left(f\left(x_{t}\right)\right) \leq \frac{f_{-}^{\prime}(M)-f_{+}^{\prime}(m)}{M-m} \phi_{t}\left(\left[M \mathbf{1}-x_{t}\right]\left[x_{t}-m \mathbf{1}\right]\right),
$$

while integrating yields

$$
\begin{aligned}
& \alpha_{f} \int_{T} \phi_{t}\left(x_{t}\right) d \mu(t)+\beta_{f} \mathbf{1}-\int_{T} \phi_{t}\left(f\left(x_{t}\right)\right) d \mu(t) \\
\leq & \frac{f_{-}^{\prime}(M)-f_{+}^{\prime}(m)}{M-m} \int_{T} \phi_{t}\left(\left[M \mathbf{1}-x_{t}\right]\left[x_{t}-m \mathbf{1}\right]\right) d \mu(t),
\end{aligned}
$$

so that the second inequality in (2.6) holds.

Taking into account Theorem 2.1, it is enough to justify the third inequality sign in (2.6). To prove our assertion, we note that the function

$$
h(t)=(M-t)(t-m)=-t^{2}+(M+m) t-M m, \quad t \in[m, M]
$$

is operator concave (see e.g. [3]). Finally, applying the Jensen operator inequality (1.1) to the above function $h$, it follows that

$$
\begin{aligned}
& \int_{T} \phi_{t}\left(\left[M \mathbf{1}-x_{t}\right]\left[x_{t}-m \mathbf{1}\right]\right) d \mu(t) \\
\leq & \left(M \mathbf{1}-\int_{T} \phi_{t}\left(x_{t}\right) d \mu(t)\right)\left(\int_{T} \phi_{t}\left(x_{t}\right) d \mu(t)-m \mathbf{1}\right),
\end{aligned}
$$

and the proof is completed.

Below, series of inequalities in (2.1) and (2.6) will be applied to quasi-arithmetic and power operator means.

\section{Applications to quasi-arithmetic operator means}

Roughly speaking, an arbitrary $C^{*}$-algebra is isomorphic to a $C^{*}$-algebra of bounded operators on a Hilbert space $\mathcal{H}$, denoted by $\mathfrak{B}(\mathcal{H})$. It is a consequence of the well-known Gelfand-Naimark theorem (see [4]). Hence, for the reader convenience, from now on, $C^{*}$-algebras will be regarded as algebras of bounded operators on a Hilbert space.

Now, for the Hilbert spaces $\mathcal{H}$ and $\mathcal{K}$, let $P[\mathfrak{B}(\mathcal{H}), \mathfrak{B}(\mathcal{K})]$ denotes the set of all fields $\left(\phi_{t}\right)_{t \in T}$ of positive linear mappings $\phi_{t}: \mathfrak{B}(\mathcal{H}) \rightarrow \mathfrak{B}(\mathcal{K})$, defined on a locally compact Hausdorff space $T$ with a bounded Radon measure $\mu$, which are unital.

A generalized quasi-arithmetic operator mean is defined by

$$
M_{\psi}(x, \phi)=\psi^{-1}\left(\int_{T} \phi_{t}\left(\psi\left(x_{t}\right)\right) d \mu(t)\right),
$$

where $\left(x_{t}\right)_{t \in T}$ is a continuous field of operators in $\mathfrak{B}(\mathcal{H})$ with spectra in $[m, M] \subseteq \mathbb{R}$, $\left(\phi_{t}\right)_{t \in T} \in P[\mathfrak{B}(\mathcal{H}), \mathfrak{B}(\mathcal{K})]$, and $\psi:[m, M] \rightarrow \mathbb{R}$ is a continuous strictly monotone function.

Throughout this section we also use the notation

$$
\psi_{m}=\min \{\psi(m), \psi(M)\}, \quad \psi_{M}=\max \{\psi(m), \psi(M)\},
$$

for a continuous strictly monotone function $\psi:[m, M] \rightarrow \mathbb{R}$. 
In paper [9], Mićić et.al. investigated an order among the above quasi-arithmetic means. More precisely, they obtained that the inequality

$$
M_{\psi}(x, \phi) \leq M_{\chi}(x, \phi)
$$

holds if one of the following two conditions is fulfilled:

(i) $\chi \circ \psi^{-1}$ is operator convex and $\chi^{-1}$ is operator monotone,

(ii) $\chi \circ \psi^{-1}$ is operator concave and $-\chi^{-1}$ is operator monotone.

On the other hand, if

(i') $\chi \circ \psi^{-1}$ is operator concave and $\chi^{-1}$ is operator monotone,

(ii') $\chi \circ \psi^{-1}$ is operator convex and $-\chi^{-1}$ is operator monotone, then the sign of inequality in (3.2) is reversed.

Moreover, if $\psi^{-1}$ is operator convex and $\chi^{-1}$ is operator concave, then

$$
M_{\psi}(x, \phi) \leq M_{1}(x, \phi) \leq M_{\chi}(x, \phi),
$$

while for operator concave function $\psi^{-1}$ and operator convex function $\chi^{-1}$ the signs of inequalities in series (3.3) are reversed.

As we see, the above relations (3.2) and (3.3), regarding order among quasi-arithmetic means, are derived via operator convexity and operator monotonicity. For more details about an order among operator means, the reader is referred to papers [7], [8] and [9].

As distinguished from the above relations (3.2) and (3.3), converses of quasi-arithmetic operator means are derived by virtue of the convexity and monotonicity in the classical real sense. The corresponding result can be carried out by virtue of our Theorem 2.1.

3.1. Theorem. Let $\chi, \psi: I \rightarrow \mathbb{R}$ be continuous strictly monotone functions and let the interval $[m, M]$ belongs to the interior of interval I. Further, suppose that $\chi \circ \psi^{-1}$ is well-defined and convex on $\psi(I)$. If $\left(\phi_{t}\right)_{t \in T} \in P[\mathfrak{B}(\mathcal{H}), \mathfrak{B}(\mathcal{K})]$, where $\mathcal{H}, \mathcal{K}$ are Hilbert spaces and $T$ is a locally compact Hausdorff space with a bounded Radon measure $\mu$, then the series of inequalities

$$
\begin{aligned}
\chi & \left(M_{\chi}(x, \phi)\right)-\chi\left(M_{\psi}(x, \phi)\right) \\
\leq & \frac{\left(\chi \circ \psi^{-1}\right)_{-}^{\prime}\left(\psi_{M}\right)-\left(\chi \circ \psi^{-1}\right)_{+}^{\prime}\left(\psi_{m}\right)}{\psi_{M}-\psi_{m}}\left[\psi_{M} \mathbf{1}-\psi\left(M_{\psi}(x, \phi)\right)\right] \\
& \times\left[\psi\left(M_{\psi}(x, \phi)\right)-\psi_{m} \mathbf{1}\right] \\
\leq & \frac{1}{4}\left(\psi_{M}-\psi_{m}\right)\left[\left(\chi \circ \psi^{-1}\right)_{-}^{\prime}\left(\psi_{M}\right)-\left(\chi \circ \psi^{-1}\right)_{+}^{\prime}\left(\psi_{m}\right)\right] \mathbf{1}
\end{aligned}
$$

holds for every continuous field $\left(x_{t}\right)_{t \in T}$ of operators in $\mathfrak{B}(\mathcal{H})$ with spectra in $[m, M]$. Further, if $\chi \circ \psi^{-1}$ is concave on $\psi(I)$, then the signs of inequalities in (3.4) are reversed.

Proof. Since $\psi: I \rightarrow \mathbb{R}$ is a continuous strictly monotone function, it follows that $\psi_{m} \leq$ $\psi(t) \leq \psi_{M}$, for all $t \in[m, M]$. Moreover, by virtue of the functional calculus, it follows that $\psi_{m} \mathbf{1} \leq \psi\left(x_{t}\right) \leq \psi_{M} \mathbf{1}$ for every $t \in T$. This means that the spectra of the field $\left(y_{t}\right)_{t \in T}=\left(\psi\left(x_{t}\right)\right)_{t \in T}$ is contained in the interval $\left[\psi_{m}, \psi_{M}\right]$.

On the other hand, since the function $\chi \circ \psi^{-1}$ is obviously continuous on $\psi(I)$, the interval $\left[\psi_{m}, \psi_{M}\right]$ belongs to the interior of $\psi(I)$.

Finally, utilizing Theorem 2.1, that is, the series of inequalities in (2.1) with $\psi_{m}, \psi_{M}$, $\chi \circ \psi^{-1},\left(y_{t}\right)_{t \in T}$ respectively instead of $m, M, f,\left(x_{t}\right)_{t \in T}$, and with definition (3.1) of quasi-arithmetic means, we obtain (3.4).

3.2. Remark. Clearly, with assumptions as in Theorem 3.1 , the operator $\chi\left(M_{\chi}(x, \phi)\right)-$ $\chi\left(M_{\psi}(x, \phi)\right)$ is not positive in general. It is positive if the function $\chi \circ \psi^{-1}$ is operator convex on the corresponding interval. Moreover, applying operator convexity and monotonicity to suitable functions, one obtains relations (3.2) and (3.3). For more details the reader is referred to [9]. 
With the same setting as in the previous result, Theorem 2.4 can also be exploited in deriving converses of the Lah-Ribarič operator inequality involving quasi-arithmetic means.

3.3. Theorem. Let $\chi, \psi: I \rightarrow \mathbb{R}$ be continuous strictly monotone functions and let the interval $[m, M]$ belongs to the interior of interval I. Further, suppose that $\chi \circ \psi^{-1}$ is well-defined and convex on $\psi(I)$. If $\left(\phi_{t}\right)_{t \in T} \in P[\mathfrak{B}(\mathcal{H}), \mathfrak{B}(\mathcal{K})]$, where $\mathcal{H}, \mathcal{K}$ are Hilbert spaces and $T$ is a locally compact Hausdorff space with a bounded Radon measure $\mu$, then the series of inequalities

$$
\begin{aligned}
0 \leq & \frac{\chi(M)-\chi(m)}{\psi(M)-\psi(m)} \psi\left(M_{\psi}(x, \phi)\right)+\frac{\psi(M) \chi(m)-\psi(m) \chi(M)}{\psi(M)-\psi(m)} \mathbf{1}-\chi\left(M_{\chi}(x, \phi)\right) \\
\leq & \frac{\left(\chi \circ \psi^{-1}\right)_{-}^{\prime}\left(\psi_{M}\right)-\left(\chi \circ \psi^{-1}\right)_{+}^{\prime}\left(\psi_{m}\right)}{\psi_{M}-\psi_{m}} \\
& \times \int_{T} \phi_{t}\left(\left[\psi_{M} \mathbf{1}-\psi\left(x_{t}\right)\right]\left[\psi\left(x_{t}\right)-\psi_{m} \mathbf{1}\right]\right) d \mu(t) \\
\leq & \frac{\left(\chi \circ \psi^{-1}\right)_{-}^{\prime}\left(\psi_{M}\right)-\left(\chi \circ \psi^{-1}\right)_{+}^{\prime}\left(\psi_{m}\right)}{\psi_{M}-\psi_{m}}\left[\psi_{M} \mathbf{1}-\psi\left(M_{\psi}(x, \phi)\right)\right] \\
& \times\left[\psi\left(M_{\psi}(x, \phi)\right)-\psi_{m} \mathbf{1}\right] \\
\leq & \frac{1}{4}\left(\psi_{M}-\psi_{m}\right)\left[\left(\chi \circ \psi^{-1}\right)_{-}^{\prime}\left(\psi_{M}\right)-\left(\chi \circ \psi^{-1}\right)_{+}^{\prime}\left(\psi_{m}\right)\right] \mathbf{1}
\end{aligned}
$$

holds for every continuous field $\left(x_{t}\right)_{t \in T}$ of operators in $\mathfrak{B}(\mathcal{H})$ with spectra in $[m, M]$. If $\chi \circ \psi^{-1}$ is concave on $\psi(I)$, then the signs of inequalities in (3.5) are reversed.

Proof. Considering the same setting as in the proof of Theorem 3.1 and with notation as in Theorem 2.4, we have

$$
\alpha_{\chi \circ \psi^{-1}}=\frac{\chi(M)-\chi(m)}{\psi(M)-\psi(m)}, \quad \beta_{\chi \circ \psi^{-1}}=\frac{\psi(M) \chi(m)-\psi(m) \chi(M)}{\psi(M)-\psi(m)}
$$

so the result is an immediate consequence of the series of inequalities in (2.6).

3.4. Remark. The first inequality in (3.5) can be rewritten in the following form:

$$
\begin{aligned}
& (\psi(M)-\psi(m)) \chi\left(M_{\chi}(x, \phi)\right)-(\chi(M)-\chi(m)) \psi\left(M_{\psi}(x, \phi)\right) \\
& \leq(\psi(M) \chi(m)-\psi(m) \chi(M)) \mathbf{1} .
\end{aligned}
$$

The above inequality (3.6) can be regarded as an operator analogue of the corresponding relation for linear functionals (see [10], Theorem 4.3, p. 108).

3.5. Remark. With notations as in Theorems 3.1 and 3.3 , suppose that the function $\chi \circ \psi^{-1}$ is differentiable in points $\psi_{m}$ and $\psi_{M}$. In this case expressions $\psi_{m}$ and $\psi_{M}$ in (3.4) and (3.5) can respectively be replaced by $\psi(m)$ and $\psi(M)$, due to the symmetry. In addition, utilizing a chain rule, the expression

$$
\left(\chi \circ \psi^{-1}\right)_{-}^{\prime}(\psi(M))-\left(\chi \circ \psi^{-1}\right)_{+}^{\prime}(\psi(m))
$$

can be rewritten in a more suitable form, that is,

$$
\left(\chi \circ \psi^{-1}\right)_{-}^{\prime}(\psi(M))-\left(\chi \circ \psi^{-1}\right)_{+}^{\prime}(\psi(m))=\frac{\chi^{\prime}(M)}{\psi^{\prime}(M)}-\frac{\chi^{\prime}(m)}{\psi^{\prime}(m)} .
$$




\section{Applications to power operator means}

As a particular case of a quasi-arithmetic mean defined by (3.1), we may consider a power operator mean (see e.g. [8]):

$$
M_{r}(x, \phi)= \begin{cases}\left(\int_{T} \phi_{t}\left(x_{t}^{r}\right) d \mu(t)\right)^{\frac{1}{r}}, & r \neq 0 \\ \exp \left(\int_{T} \phi_{t}\left(\log x_{t}\right) d \mu(t)\right), & r=0 .\end{cases}
$$

By virtue of relations (3.2) and (3.3), Mićić et.al. [9], established the following order among power operator means:

$$
M_{r}(x, \phi) \leq M_{s}(x, \phi),
$$

for either $r \leq s, r, s \in \mathbb{R} \backslash[-1,1]$ or $\frac{1}{2} \leq r \leq 1 \leq s$ or $r \leq-1 \leq s \leq-\frac{1}{2}$. However, a class of inequalities in (4.2) is a consequence of operator convexity and monotonicity of the corresponding power functions.

On the other hand, regarding the method developed in this paper, converses for power operator means are established via the classical convexity. The following result appears to be a consequence of Theorem 3.1 when considering the above power operator means.

4.1. Theorem. Let $\left(\phi_{t}\right)_{t \in T} \in P[\mathfrak{B}(\mathcal{H}), \mathfrak{B}(\mathcal{K})]$, where $\mathcal{H}, \mathcal{K}$ are Hilbert spaces and $T$ is a locally compact Hausdorff space with a bounded Radon measure $\mu$, and let $\left(x_{t}\right)_{t \in T}$ be a continuous field of positive operators in $\mathfrak{B}(\mathcal{H})$ with spectra in $[m, M] \subseteq \mathbb{R}_{+}$.

(i) If either $s \leq 0<r$ or $r<0 \leq s$ or $0<r<s$ or $s<r<0$, then the following series of inequalities holds:

$$
\begin{aligned}
& {\left[M_{s}(x, \phi)\right]^{s}-\left[M_{r}(x, \phi)\right]^{s} } \\
\leq & \frac{s}{r} \cdot \frac{M^{s-r}-m^{s-r}}{M^{r}-m^{r}}\left[M^{r} \mathbf{1}-\left[M_{r}(x, \phi)\right]^{r}\right]\left[\left[M_{r}(x, \phi)\right]^{r}-m^{r} \mathbf{1}\right] \\
\leq & \frac{s}{4 r}\left(M^{r}-m^{r}\right)\left(M^{s-r}-m^{s-r}\right) \mathbf{1} .
\end{aligned}
$$

Further, if $0 \leq s<r$ or $r<s \leq 0$, then the signs of inequalities in (4.3) are reversed.

(ii) If $r<0$ then

$$
\begin{aligned}
0 & \leq \log \left[M_{0}(x, \phi)\right]-\log \left[M_{r}(x, \phi)\right] \\
& \leq-\frac{1}{r M^{r} m^{r}}\left[M^{r} \mathbf{1}-\left[M_{r}(x, \phi)\right]^{r}\right]\left[\left[M_{r}(x, \phi)\right]^{r}-m^{r} \mathbf{1}\right] \\
& \leq-\frac{\left(M^{r}-m^{r}\right)^{2}}{4 r M^{r} m^{r}} \mathbf{1}
\end{aligned}
$$

while for $r>0$ the signs of inequalities in (4.4) are reversed.

(iii) If $s \in \mathbb{R}$, then the following series of inequalities holds:

$$
\begin{aligned}
& {\left[M_{s}(x, \phi)\right]^{s}-\left[M_{0}(x, \phi)\right]^{s} } \\
\leq & \frac{s\left(M^{s}-m^{s}\right)}{\log M-\log m}\left[\log M \mathbf{1}-\log \left[M_{0}(x, \phi)\right]\right]\left[\log \left[M_{0}(x, \phi)\right]-\log m \mathbf{1}\right] \\
\leq & \frac{s}{4}(\log M-\log m)\left(M^{s}-m^{s}\right) \mathbf{1} .
\end{aligned}
$$

Proof. The proof is a simple consequence of Theorem 3.1, that is, the series of inequalities in (3.4) with particular choices of functions $\chi$ and $\psi$.

More precisely, let $\chi(t)=t^{s}$ and $\psi(t)=t^{r}$, where $s$ and $r$ are mutually different real parameters not equal to zero. Then the function $\left(\chi \circ \psi^{-1}\right)(t)=t^{\frac{s}{r}}$ is convex on $\mathbb{R}_{+}$if $\frac{s}{r} \leq 0$ or $\frac{s}{r} \geq 1$. It is possible in each of the following four cases: $s<0<r$ or $r<0<s$ 
or $0<r<s$ or $s<r<0$. Finally, since $\left(\chi \circ \psi^{-1}\right)^{\prime}(t)=\frac{s}{r} t^{\frac{s-r}{r}}$, considering (3.4) with the above functions $\chi$ and $\psi$ on the interval $[m, M]$, we obtain (4.3).

On the other hand, the function $\left(\chi \circ \psi^{-1}\right)(t)=t^{\frac{s}{r}}$ is concave on $\mathbb{R}_{+}$if $0 \leq \frac{s}{r} \leq 1$, hence if $0<s<r$ or $r<s<0$ we obtain series (4.3) with reversed signs of inequalities. Clearly, the series of inequalities in (4.3), as well as the series with reversed signs of inequalities, holds also for $s=0$.

It remains to consider the cases when one of the parameters $r$ and $s$ is equal to zero. If $s=0$, then setting $\chi(t)=\log t$ and $\psi(t)=t^{r}$, it follows that $\left(\chi \circ \psi^{-1}\right)(t)=\frac{1}{r} \log t$. Clearly, this function is convex for $r<0$, while it is concave for $r>0$. Moreover, since $\left(\chi \circ \psi^{-1}\right)^{\prime}(t)=\frac{1}{r t}$, after a straightforward computation we obtain (4.4) without the first inequality sign in the convex case, while in the concave case the reversed series of inequalities holds. The first inequality sign in (4.4), as well as in the reversed series of inequalities, holds due to the operator convexity of the function $\frac{1}{r} \log t$ when $r<0$, that is, operator concavity when $r>0$.

Finally, if $r=0$, then setting $\chi(t)=t^{s}$ and $\psi(t)=\log t$, it follows that the function $\left(\chi \circ \psi^{-1}\right)(t)=\exp (s t)$ is convex for every $s \neq 0$. In addition, $\left(\chi \circ \psi^{-1}\right)^{\prime}(t)=s \exp (s t)$, which yields (4.5) after a straightforward computation. Of course, the series of inequalities in (4.5) holds also for $s=0$.

4.2. Remark. Observe that the function $\left(\chi \circ \psi^{-1}\right)(t)=\frac{1}{r} \log t$ is simultaneously convex and operator convex, that is, concave and operator concave depending on whether $r<0$ or $r>0$. Hence, the first expression in (4.4) is the positive operator yielding the inequality

$$
\log \left[M_{r}(x, \phi)\right] \leq \log \left[M_{0}(x, \phi)\right]
$$

for $r<0$. On the other hand, if $r>0$ then the following inequality holds:

$$
\log \left[M_{0}(x, \phi)\right] \leq \log \left[M_{r}(x, \phi)\right] .
$$

It is well-known that the function $f(t)=t^{r}$ is operator convex on $\mathbb{R}_{+}$if either $1 \leq$ $r \leq 2$ or $-1 \leq r \leq 0$, and is operator concave on $\mathbb{R}_{+}$when $0 \leq r \leq 1$. Hence, discussing the operator convexity of the function $\left(\chi \circ \psi^{-1}\right)(t)=t^{\frac{s}{r}}$ (see the proof of Theorem 4.1), we obtain conditions on parameters $r$ and $s$ under which the operator $\left[M_{s}(x, \phi)\right]^{s}-\left[M_{r}(x, \phi)\right]^{s}$ is positive in the series of inequalities (4.3).

4.3. Corollary. With the same assumptions as in the statement of Theorem 4.1, the series of inequalities

$$
\begin{aligned}
0 & \leq\left[M_{s}(x, \phi)\right]^{s}-\left[M_{r}(x, \phi)\right]^{s} \\
& \leq \frac{s}{r} \cdot \frac{M^{s-r}-m^{s-r}}{M^{r}-m^{r}}\left[M^{r} \mathbf{1}-\left[M_{r}(x, \phi)\right]^{r}\right]\left[\left[M_{r}(x, \phi)\right]^{r}-m^{r} \mathbf{1}\right] \\
& \leq \frac{s}{4 r}\left(M^{r}-m^{r}\right)\left(M^{s-r}-m^{s-r}\right) \mathbf{1}
\end{aligned}
$$

holds if either $0<r \leq s \leq 2 r$ or $2 r \leq s \leq r<0$ or $0 \leq s+r \leq r \neq 0$ or $0 \neq r \leq r+s \leq 0$. Further, if $0 \neq r \leq s \leq 0$ or $0 \leq s \leq r \neq 0$, then the signs of inequalities in (4.6) are reversed.

Proof. Regarding the proof of Theorem 4.1, it follows that the first inequality sign in (4.6) holds when $\left(\chi \circ \psi^{-1}\right)(t)=t^{\frac{s}{r}}$ is operator convex function. This function is operator convex if either $1 \leq \frac{s}{r} \leq 2$ or $-1 \leq \frac{s}{r} \leq 0$, that is, when either $0<r \leq s \leq 2 r$ or $2 r \leq s \leq r<0$ or $0 \leq s+r \leq r \neq 0$ or $0 \neq r \leq r+s \leq 0$. Moreover, since the operator convexity of the function $\left(\chi \circ \psi^{-1}\right)(t)=t^{\frac{s}{r}}$ implies its usual convexity, it follows that the remaining signs of inequalities in (4.6) are also valid under the above conditions.

On the other hand, function $\left(\chi \circ \psi^{-1}\right)(t)=t^{\frac{s}{r}}$ is operator concave if $0 \leq \frac{r}{s} \leq 1$, that is, when $0 \neq r \leq s \leq 0$ or $0 \leq s \leq r \neq 0$. Under these conditions $\left(\chi \circ \psi^{-1}\right)(t)=$ 
$t^{\frac{s}{r}}$ is concave in the classical sense, as well. This gives (4.6) with reversed signs of inequalities.

4.4. Remark. With the conditions as in Corollary 4.3, we obtain the order among operators $\left[M_{s}(x, \phi)\right]^{s}$ and $\left[M_{r}(x, \phi)\right]^{s}$. Moreover, applying the operator monotonicity of suitable power functions, one obtains conditions as in (4.2). In fact, it is a more specific use of relations (3.2) and (3.3), for more details see [9].

4.5. Remark. It should be noticed here that the above discussion as in Corollary 4.3 and Remark 4.2 can not be applied to the series of inequalities in (4.5) since the exponential function $f(t)=\exp t$ is not operator convex (see e.g. [1]).

Guided by the proof of Theorem 4.1, we also obtain an interesting consequence of Theorem 3.3, that is, the converses of the Lah-Ribarič inequality that correspond to power operator means.

4.6. Theorem. Let $\left(\phi_{t}\right)_{t \in T} \in P[\mathfrak{B}(\mathcal{H}), \mathfrak{B}(\mathcal{K})]$, where $\mathcal{H}, \mathcal{K}$ are Hilbert spaces and $T$ is a locally compact Hausdorff space with a bounded Radon measure $\mu$, and let $\left(x_{t}\right)_{t \in T}$ be a continuous field of positive operators in $\mathfrak{B}(\mathcal{H})$ with spectra in $[m, M] \subseteq \mathbb{R}_{+}$.

(i) If either $s \leq 0<r$ or $r<0 \leq s$ or $0<r<s$ or $s<r<0$, then the following series of inequalities holds:

$$
\begin{aligned}
0 & \leq \frac{M^{s}-m^{s}}{M^{r}-m^{r}}\left[M_{r}(x, \phi)\right]^{r}+\frac{M^{r} m^{s}-m^{r} M^{s}}{M^{r}-m^{r}} \mathbf{1}-\left[M_{s}(x, \phi)\right]^{s} \\
& \leq \frac{s}{r} \cdot \frac{M^{s-r}-m^{s-r}}{M^{r}-m^{r}} \int_{T} \phi_{t}\left(\left[M^{r} \mathbf{1}-x_{t}^{r}\right]\left[x_{t}^{r}-m^{r} \mathbf{1}\right]\right) d \mu(t) \\
& \leq \frac{s}{r} \cdot \frac{M^{s-r}-m^{s-r}}{M^{r}-m^{r}}\left[M^{r} \mathbf{1}-\left[M_{r}(x, \phi)\right]^{r}\right]\left[\left[M_{r}(x, \phi)\right]^{r}-m^{r} \mathbf{1}\right] \\
& \leq \frac{s}{4 r}\left(M^{r}-m^{r}\right)\left(M^{s-r}-m^{s-r}\right) \mathbf{1} .
\end{aligned}
$$

Moreover, if $0 \leq s<r$ or $r<s \leq 0$, then the signs of inequalities in (4.7) are reversed.

(ii) If $r<0$ then

$$
\begin{aligned}
0 & \leq \frac{\log M-\log m}{M^{r}-m^{r}}\left[M_{r}(x, \phi)\right]^{r}+\frac{M^{r} \log m-m^{r} \log M}{M^{r}-m^{r}} \mathbf{1}-\log \left[M_{0}(x, \phi)\right] \\
& \leq-\frac{1}{r M^{r} m^{r}} \int_{T} \phi_{t}\left(\left[M^{r} \mathbf{1}-x_{t}^{r}\right]\left[x_{t}^{r}-m^{r} \mathbf{1}\right]\right) d \mu(t) \\
& \leq-\frac{1}{r M^{r} m^{r}}\left[M^{r} \mathbf{1}-\left[M_{r}(x, \phi)\right]^{r}\right]\left[\left[M_{r}(x, \phi)\right]^{r}-m^{r} \mathbf{1}\right] \\
& \leq-\frac{\left(M^{r}-m^{r}\right)^{2}}{4 r M^{r} m^{r}} \mathbf{1},
\end{aligned}
$$

while for $r>0$ the signs of inequalities in (4.8) are reversed.

(iii) The series of inequalities

$$
\begin{aligned}
0 & \leq \frac{M^{s}-m^{s}}{\log M-\log m} \log \left[M_{0}(x, \phi)\right]+\frac{m^{s} \log M-M^{s} \log m}{\log M-\log m} \mathbf{1}-\left[M_{s}(x, \phi)\right]^{s} \\
& \leq \frac{s\left(M^{s}-m^{s}\right)}{\log M-\log m} \int_{T} \phi_{t}\left(\left[\log M \mathbf{1}-\log x_{t}\right]\left[\log x_{t}-\log m \mathbf{1}\right]\right) d \mu(t) \\
& \leq \frac{s\left(M^{s}-m^{s}\right)}{\log M-\log m}\left[\log M \mathbf{1}-\log \left[M_{0}(x, \phi)\right]\right]\left[\log \left[M_{0}(x, \phi)\right]-\log m \mathbf{1}\right] \\
& \leq \frac{s}{4}(\log M-\log m)\left(M^{s}-m^{s}\right) \mathbf{1} \\
& \text { holds for all } s \in \mathbb{R} .
\end{aligned}
$$


Proof. We use the same procedure as in the proof of Theorem 4.1, applied to the series of inequalities in (3.5).

\section{References}

[1] R. Bhatia, Matrix Analysis, Springer-Verlag, 1997.

[2] S.S. Dragomir, Reverses of the Jensen's inequality in terms of first derivative and applications, RGMIA Res. Rep. Coll. (2011), preprint.

[3] T. Furuta, J. Mićić Hot, J. Pečarić, Y. Seo, Mond-Pečarić Method in Operator Inequalities, Element, Zagreb, 2005.

[4] I. Gelfand, M. Naimark, On the imbedding of normed rings into the ring of operators on a Hilbert space, Math. Sbornik 12 (1943), 197-217.

[5] F. Hansen, G. Pedersen, Jensen's operator inequality, Bull. London Math. Soc. 35 (2003), $553-564$.

[6] F. Hansen, J. Pečarić, I. Perić, Jensen's operator inequality and its converses, Math. Scand. 100 (2007), 61-73.

[7] J. Mićić, J. Pečarić, Order among power means of positive operators, II, Sci. Math. Japon. 71 (2010), 93-109.

[8] J. Mićić, J. Pečarić, Y. Seo, Converses of Jensen's operator inequality, Oper. Matrices 4 (2010), 385-403.

[9] J. Mićić, J. Pečarić, Y. Seo, Order among quasi-arithmetic means of positive operators, Math. Reports, in press.

[10] J.E. Pečarić, F. Proschan, Y.L. Tong, Convex functions, partial orderings and statistical applications, Academic Press Inc., San Diego, 1992. 
